Dr. med.

Brigitte Moreano

Stellvertretende

Chefredakteurin

brigitte.moreano@

springer.com

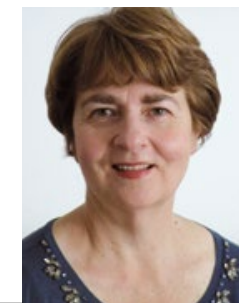

Möglicher Zusammenhang?

\section{Erst Koloskopie, dann Appendizitis}

Stehen scheint im Beruf noch ungünstiger als Sitzen zu sein. In einer kanadischen Studie wurden Daten von 7.320 zunächst herzgesunden Beschäftigten analysiert. Im Laufe von zwölf Jahren wurde bei 3,4\% der Teilnehmer eine Herzerkrankung (Infarkt oder Insuffizienz) diagnostiziert. Gegenüber einer überwiegend sitzenden Tätigkeit erhöhte dauerndes Stehen das Risiko für eine Herzkrankheit auf das Doppelte. Ein Wechsel von Stehen, Sitzen und Gehen während der Arbeit wirkte bei Männern dagegen protektiv; bei ihnen halbierte sich das kardiale Risiko.

Setzen bitte!

- Am J Epidemiol 2017, online 22. September 2017

\title{
Metaanalyse
}

\section{Kreuzbandplastik: je früher, desto besser}

Bei einem Riss des vorderen Kreuzbandes sollte die Operation nicht zu lange hinausgezögert werden, um das Arthroserisiko möglichst gering zu halten. Das ergab die Metaanalyse von 38 Studien mit mehr als 4.000 Patienten. Für das Risiko einer Knierarthrose in den folgenden Jahren spielte es eine wichtige Rolle, wie viel Zeit zwischen dem Kreuzbandriss und dem Eingriff verstrichen war. Nach einem Intervall von sechs Monaten lag die Arthroserate bei $11,7 \%$, nach 18 Monaten bei 21,6\% und nach 36 Monaten bei 45,3\%.

- Am J Sports Med 2017, online 6. Oktober

\section{Transfusionsmedizin}

\section{Mütterblut fatal für Männer?}

Für männliche Transfusionspatienten könnte es von Nachteil sein, wenn sie Erythrozytenkonzentrat erhalten, das von einer Frau stammt, die schon einmal schwanger war: Laut einer großen retrospektiven Analyse haben sie eine signifikant höhere Mortalität als Männer, bei denen eine Frau ohne frühere Schwangerschaft oder ein Mann als Blutspender fungiert haben.

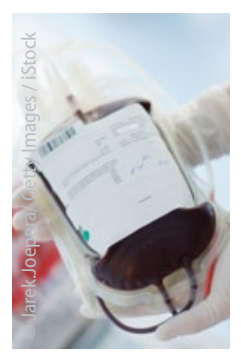

Ausgewertet wurden Daten von 31.118 Transfusionspatienten, die jeweils nur Blut von einem der drei Spendertypen - männlich, weiblich mit und weiblich ohne frühere Schwangerschaft - bekommen hatten. Als mögliche Erklärung für die erstaunliche Beobachtung vermuten die Autoren immunologische Prozesse in der Schwangerschaft. - JAMA 2017;318(15):1471-1478
Wenn Patienten nach einer Koloskopie über anhaltende Schmerzen im rechten unteren Quadranten klagen, sollte man vermehrt an eine mögliche Appendizitis denken, raten US-amerikanische Ärzte. Sie hatten Daten von über 390.000 ehemaligen Soldaten ausgewertet, die sich einer Screeningkoloskopie unterzogen hatten. Dabei stellten sie fest, dass das Risiko für eine Entzündung der Appendix in der ersten Woche nach einer Darmspiegelung deutlich höher ist als in der Zeit danach. Eine Appendektomie mit Appendizitisdiagnose hatten in der ersten Woche nach der Untersuchung zwölf Patienten, in den 51 Wochen danach $79 \mathrm{~Pa}$ tienten erhalten. Das entsprach einem Inzidenzverhältnis von 6,8. Die Zahlen sprechen nach Ansicht der Autoren dafür, dass „eine Darmspiegelung für das Auftreten einer Appendizitis innerhalb von einer Woche prädisponieren kann“.

JAMA Surgery 2017, online 4. Oktober 2017

\section{Bündel aus 6 CME-Modulen - für Sie geschnürt}

In der kommenden Ausgabe (Sonderheft 2/ 2017), die schon nächste Woche erscheint, haben Sie wieder Gelegenheit, Ihr Fortbildungskonto aufzufüllen. Sechsmal 2 Punkte erwarten Sie, wenn Sie sich zu folgenden Themen informieren:

- Aktuelle Therapieoptionen bei COPD

- Neues in der Therapie der Hepatitis C

- Opioide in der Hausarztpraxis

- Was steckt hinter dem roten Auge?

- Orale Antikoagulation bei Vorhofflimmern und

- Akutbehandlung beim allergischen Schock. 